\title{
Space-Time versus World-Sheet Renormalization Group Equation in String Theory
}

\author{
Ram Brustein ${ }^{1}$ \\ Theory Group, Dept. of Physics, \\ University of Texas, Austin Tx 78712 \\ Kaj Roland ${ }^{1,2}$ \\ Niels Bohr Institute, \\ Blegdamsvej 17, DK-2100 København Ø, Denmark
}

\begin{abstract}
We discuss the relation between space-time renormalization group equation for closed string field theory and world-sheet renormalization group equation for first-quantized strings. Restricting our attention to massless states we argue that there is a one-to-one correspondence between the fixed point solutions of the two renormalization group equations. In particular, we show how to extract the Fischler-Susskind mechanism from the string field theory equation in the case of the bosonic string.
\end{abstract}

${ }^{1}$ Supported in part by Robert A. Welch Foundation and NSF grant PHY 9009850

${ }^{2}$ Supported in part by the Danish Natural Science Research Council and the SARC Foundation. 


\section{Introduction}

In the quest for a non-perturbative formulation of string theory matrix models have been the most exciting development so far. Matrix models are characterized by differential equations, the string and flow equations [1]. For a recent review see [2]. The solution of these equations gives the vacuum energy of the theory as a function of the coupling constants and hence, in principle, specifies the value of the couplings corresponding to the configuration of least energy. The string and flow equations are known to be related to factorization properties of perturbative string amplitudes $[3,4]$. They contain information from the boundaries of moduli space only [5].

The continuum limit of matrix models are non-critical string theories. However, many of these non-critical string theories are really critical string theories in a background (see e.g. [6] ). We therefore expect the basic equations for non-critical and critical low dimensional string theories to be similar.

Based on this evidence it has been suggested that similar equations with potentially non-perturbative content may be derived for closed string theories in the critical dimension (where a non-perturbative formulation is much more complicated) by considering factorization of the perturbative amplitudes defined by the Polyakov path integral [7].

A natural framework for such considerations is the closed string field theory (SFT) developed in $[8,9,10]$. The theory is constructed from off-shell factorizable scattering amplitudes in a given background. The background can in principle be non-flat, even though the constructions of refs. $[8,9,10]$ were carried out in a flat background. As shown in ref.[11], it is possible to obtain an analogue of the Polchinski renormalization group ( $R G$ ) equation [12], for closed SFT. It is a space-time RG equation, by which we mean that it is an equation describing the dependence of the SFT action on an ultraviolet space-time cut-off. This RG equation consists of an infinite set of recursion relations encoding all factorization properties of first quantized amplitudes as well as all the information about the gauge invariance in string theory $[8,11]$. It can be hoped that these equations in critical string theory contains information similar to that of the string and flow equations in the matrix models.

On the other hand, it is well known that the factorization properties of first quantized amplitudes encoded in the SFT RG equation are also related to a world- 
sheet $\mathrm{RG}$ equation, that is, an RG equation involving the running of the background fields in the 2-dimensional sigma model w.r.t. the world sheet cut-off [13]. More specifically, on a higher genus Riemann surface the factorization properties enables one to identify non-vanishing tadpoles that can be removed by a shift of the background according to the mechanism of Fischler and Susskind [14]. The fixed point equation for the world-sheet RG equation is identical to space-time equations of motion for the background fields [15]. For a review and an extensive list of references on world-sheet RG equation, see ref.[16].

The above considerations suggest a deep connection between the space-time and the world-sheet RG equations and their fixed point solutions. In the present paper we investigate this connection by considering the "infrared" limit of the SFT RG equation, where all massive states are integrated out. We consider first a flat background, then a curved background to leading order in the background curvature and in the tadpoles. In these cases we prove that the fixed point condition for the space-time RG equation coincides with the fixed point condition for the world-sheet $\mathrm{RG}$ equation and therefore also with space-time equations of motion.

The equations of motion for the background do not fix the dilaton potential (or in general, the effective action) as a function of the string coupling constant. Rather they fix the shape of the background consistent with whatever potential is chosen. We still have to rely on the (non-Borel summable) perturbation series to determine the correct dilaton potential. However, the SFT RG equation contains much more information than just the equations of motion in the infrared limit. Therefore, the possibility that SFT RG specifies the non-perturbative string theory in full is not ruled out by the above evidence. It is possible that the study of similar RG equations in matrix models [17] can provide insight and aid in actually solving for the dilaton potential and other interesting physical quantities.

The paper is organized as follows: In section 2 we write down the space-time RG equation for string field theory and fix our notations. In section 3 we establish the connection between space-time RG equation and world-sheet RG equation in a general background. In section 4 we consider the infrared limit and discuss the fixed point solutions in flat space time, and in section 5 we generalize the discussion to non-flat and cutoff dependent backgrounds. Section 6 contains our conclusions. 


\section{Space-time RG Equation for String Field The- ory}

Consider the closed bosonic string propagating in an arbitrary 26-dimensional background specified by a generalized sigma model. In order to have well-defined scattering amplitudes we assume that the background is asymptotically flat at spatial and timelike infinity. We consider a fixed (classical) 2-dimensional metric. String field theory rewrites the first-quantized perturbation series (extended off-shell in a way consistent with factorization) in terms of vertices and propagators defining an underlying (gauge-fixed) string field theory action.

Whereas the open bosonic string allows a description in terms of a string field theory with only a cubic interaction vertex [18], the situation is considerably more complicated for the closed string. At tree level (i.e. on the sphere with genus $G=0$ ) an N-point contact term $\mathcal{V}^{0, N}$ has to be introduced. It is defined as the integral over that region of $\mathrm{N}$-punctured moduli space not covered sewing vertices with $\mathrm{N}-1$ punctures or less. The situation resembles an expansion of the Einstein-Hilbert action $\int \sqrt{-g} R$ around a fixed background.

At string loop level additional vertices $\mathcal{V}^{G, N}$ are necessary. Since the vertices represent off-shell string amplitudes they are only well-defined once a choice of world-sheet metric has been found. The actual inductive construction of these vertices has been carried out by Sonoda and Zwiebach [8] in the case of a flat background.

One arrives at a string field theory action $S$ (in real Minkowskian time) given by

$$
\begin{aligned}
i S & =-\frac{1}{2} \Psi^{A} \Delta_{A B}^{-1} \Psi^{B}+S_{i n t} \\
S_{i n t}(\Psi) & =\sum_{G, N} \frac{1}{N !} \mathcal{V}_{A_{1}, \ldots, A_{N}}^{G, N} \Psi^{A_{1}} \ldots \Psi^{A_{N}}
\end{aligned}
$$

(The choice of $S_{\text {int }}$ to be imaginary is purely notational). The string field $\Psi$ carries an index $A$ running over all BRST cohomology classes of string states (including the momentum degrees of freedom). $S$ is the gauge fixed string field action. In the gauge $\left(b_{0}+\bar{b}_{0}\right) \mid A>=0$, a general string state is of the form

$$
\left|A>=\left(A_{1}+\left(c_{0}-\bar{c}_{0}\right) A_{2}\right)\right| q=1, \bar{q}=1>
$$


where $\mid q=1, \bar{q}=1>$ is the ghost vacuum in the notation of ref.[19] and the operators $A_{1}$ and $A_{2}$ do not contain the ghost oscillators $b_{0}, \bar{b}_{0}, c_{0}, \bar{c}_{0}$.

String states are related to vertex operators by $\left|A>=\Phi_{A}(0)\right| 0>$ where $\mid 0>$ is the conformal vacuum. The innerproduct is defined by $[21,22]$

$$
\gamma_{A B} \equiv \lim _{\epsilon \rightarrow 0}\left(\frac{-1}{\epsilon^{2}}\right)^{\Delta_{A}}<\Phi_{A}\left(\frac{1}{\epsilon}\right) \Phi_{B}(0)>
$$

where $\Delta_{A}$ is the conformal weight of the state $\mid A>\cdot \gamma_{A B}$ is a metric in the space of string states and can be used to raise and lower string state indices in the usual way. Dual string states are defined by

$$
\left|A^{*}>=\gamma^{A B}\right| B>
$$

The propagator is defined as the Polyakov path integral over the cylinder, with the Schwinger proper time coordinate of the cylinder integrated over a fixed range of values, $0 \leq \tau_{1}<\tau<\tau_{2} \leq \infty$. In the gauge (2) the propagator of closed string field theory is given by

$$
\begin{aligned}
\Delta^{A B} & =-i \frac{\alpha^{\prime}}{4 \pi} \int_{\epsilon}^{a} \frac{d^{2} k}{k \bar{k}}<A^{*}\left|\bar{b}_{0} b_{0} k^{L_{0}} \bar{k}^{\bar{L}_{0}}\right| B^{*}> \\
& =-i \frac{\alpha^{\prime}}{2} \int_{\epsilon}^{a} \frac{d|k|}{|k|}<\left.A^{*}\left|\bar{b}_{0} b_{0}\right| k\right|^{L_{0}+\bar{L}_{0}} \delta_{L_{0}, \bar{L}_{0}} \mid B^{*}>
\end{aligned}
$$

where $\epsilon$ is the short distance cut-off on the world sheet and $a$ is the fixed upper limit of the sewing parameter. Obviously $0 \leq \epsilon \leq a \leq 1$. In terms of Schwinger proper time $\tau_{2}=-\log \epsilon$ and $\tau_{1}=-\log a$, and

$$
\Delta^{A B}=-i \frac{\alpha^{\prime}}{2} \int_{-\log a}^{-\log \epsilon} d \tau<A^{*}\left|\bar{b}_{0} b_{0} e^{-\tau\left(L_{0}+\bar{L}_{0}\right)} \delta_{L_{0}, \bar{L}_{0}}\right| B^{*}>
$$

If we expand SFT around flat space and use a basis of states that diagonalize the propagator then $L_{0}+\bar{L}_{0} \sim p_{A}^{2}+m_{A}^{2}$, where $p$ is space-time momentum (see eqs.(21),(25)). We therefore learn from eq.(6) that both $\log \epsilon$ and $\log a$ are associated with space-time scales. $\log \epsilon$ is related to low (infrared) momenta and $\log a$ is related to high (ultraviolet) momenta. In an ordinary field theory $-\sqrt{\alpha^{\prime}} \log a$ could actually be identified with a short distance cutoff.

The value of $a$ determines what are the interactions and what is the propagator in the theory. In the limit $a \rightarrow \epsilon$ the propagator vanishes; the vertex $\mathcal{V}_{A_{1}, \ldots, A_{N}}^{G, N}$ covers all of $N$-punctured $G$-loop moduli space with cut-off $\epsilon$ and hence represents the entire 
bare, truncated, connected $G$-loop $N$-point Green's function, $T^{G, N}\left(A_{1}, \ldots, A_{N}\right)$, computed in the chosen background. The notation in eq.(1) is chosen such that

$$
\left.i^{N} \mathcal{V}_{A_{1}, \ldots, A_{N}}^{G, N}\right|_{a=\epsilon} \equiv T^{G, N}\left(A_{1}, \ldots, A_{N}\right)
$$

We introduce the notation

$$
T^{N}=\sum_{G} T^{G, N} \quad \mathcal{V}^{N}=\sum_{G} \mathcal{V}^{G, N}
$$

On-shell, $T^{N}$ is the bare connected S-matrix element. Our conventions for the S-matrix are those of ref.[23].

By construction the propagator and the vertices all depend on $a$ but in such a way that the full connected Green's function, obtained by the integral over all of moduli space, does not. (Except, of course, for $N=2, G=0$ where we obtain the inverse of the propagator (6)). The renormalization group equation is just the statement that the functional $W$, generating these Green's functions, does not depend on $a$ (apart from the abovementioned a-dependence entering into the kinetic piece of the SFT action). Since $a$ is associated with a space-time scale this RG equation is a space-time RG equation.

Formally carrying out the path integral over string fields, the generating functional is given by

$$
W[J]=\exp \left\{S_{\text {int }}\left(\frac{\delta}{\delta J}\right)\right\} \exp \left\{\frac{1}{2} J_{A} \Delta^{A B} J_{B}\right\}
$$

As shown in ref.[11], $d W[0] / d \log a=0$ is ensured by requiring the Polchinski RG equation (adapted for SFT)

$$
\frac{\partial}{\partial \log a} S_{\text {int }}[\Psi]=-\frac{1}{2}\left(\frac{\delta S_{\text {int }}}{\delta \Psi^{A}} \frac{\delta S_{\text {int }}}{\delta \Psi^{B}}+\frac{\delta^{2} S_{\text {int }}}{\delta \Psi^{A} \delta \Psi^{B}}\right) \frac{\partial}{\partial \log a} \Delta^{A B}
$$

Singling out the piece proportional to $\Psi^{A_{1}} \ldots \Psi^{A_{N}}$ we arrive at the recursion relations [11].

$$
\begin{aligned}
0= & \sum_{G} \frac{\partial}{\partial \log a} \mathcal{V}_{A_{1}, \ldots, A_{N}}^{G, N} \\
& +\frac{1}{2}\left(\frac{\partial}{\partial \log a} \Delta^{A B}\right) \sum_{G_{1}, G_{2}} \sum_{\substack{N_{1}+N_{2} \\
=N+2}} \sum_{\substack{S_{N} \\
S_{N_{1}-1} \otimes S_{N_{2}-1}}} \mathcal{V}_{A A_{p(1)} \ldots A_{p\left(N_{1}-1\right)}}^{G_{1}, N_{1}} \mathcal{V}_{A_{p\left(N_{1}\right)}, \ldots A_{p(N)} B}^{G_{2}, N_{2}} \\
& +\frac{1}{2}\left(\frac{\partial}{\partial \log a} \Delta^{A B}\right) \sum_{G} \mathcal{V}_{A_{1}, \ldots, A_{N} A B}^{G, N+2}
\end{aligned}
$$


The vertex $\mathcal{V}_{A_{1}, \ldots, A_{N}}^{G, N}$ is defined to be symmetric in all string state indices. The summation over permutations in the second term is there to explicitly symmetrize in the indices $A_{1}, \ldots, A_{N}$. The different terms correspond to the different channels from which a higher point function can be sewn.

If we expand string field theory around a background with a constant dilaton mode we can also determine the dependence of various quantities on the string coupling constant, $\lambda$. In that case we have $\mathcal{V}^{G, N} \propto \lambda^{2 G-2+N}$ and by comparing coefficients in eq.(11) order by order in $\lambda$ we obtain $[8,11]$

$$
\begin{aligned}
0= & \frac{\partial}{\partial \log a} \mathcal{V}_{A_{1}, \ldots, A_{N}}^{G, N} \\
& +\frac{1}{2}\left(\frac{\partial}{\partial \log a} \Delta^{A B}\right) \sum_{\substack{G_{1}+G_{2} \\
=G=N+2}} \sum_{\substack{N_{1}+N_{2} \\
=G \in}} \sum_{\frac{S_{N}}{S_{N_{1}-1} \otimes S_{N_{2}-1}}} \mathcal{V}_{A A_{p(1)} \ldots A_{p\left(N_{1}-1\right)}}^{G_{1}, N_{1}} \mathcal{V}_{A_{p\left(N_{1}\right) \ldots A_{p(N)} B}^{G_{2}, N_{2}}} \\
& +\frac{1}{2}\left(\frac{\partial}{\partial \log a} \Delta^{A B}\right) \mathcal{V}_{A_{1}, \ldots, A_{N} A B}^{G-1, N+2}
\end{aligned}
$$

The RG equation constrains the form of the vertices appearing in the string field theory. As pointed out in ref.[24] it is a universal consistency condition for Riemann surfaces with coordinates.

The introduction of the vertices $\mathcal{V}^{G, N}$ correspond to a cell-decomposition of genus G moduli space. The recursion relation (12) states that all "internal" boundary contributions introduced by this decomposition vanish. Hence, full on-shell BRST invariance is satisfied if one considers the theory to be defined in terms of compactified moduli space. This space, which includes the degeneration points, by definition has no boundaries.

Full on-shell BRST invariance means that the physical content of the gauge fixed string field theory (i.e. the correlators of gauge-independent dynamical variables) does not depend on the gauge choice. Formally this is expressed by the requirement that the gauge fixed string field theory action satisfies the full Batalin-Vilkovisky master equation. Indeed, the RG equation (10) is reduced for $a=1$ to the BatalinVilkovisky equation [11, 24]. It therefore contains all the information about the gauge invariance of closed string field theory. From this point of view the recursion relations $(11,12)$ should be thought of as Ward identities for the theory.

Eqs.(9-11) are non-perturbative equations relating the running of the full quantum N-point vertices to the vertices themselves. They do not determine the vertices uniquely. From a perturbative point of view this is obvious: Although the equation 
encodes all factorization properties we still have to specify the "building blocks" of factorization, i.e. the two- and three-point functions at tree level. However, this does not mean that eqs.(9-11) could not specify the underlying SFT in a unique way. We should keep in mind that eqs.(9-11) are equations for SFT expanded around a certain classical string field configuration, specified in perturbation theory by all the background fields entering into the sigma model. Certainly the vertices $\nu^{G, N}$ will depend on the choice of this background. However, this dependence does not reflect any ambiguity on part of the SFT, only the ambiguity of choosing the background. If the choice of background is the only information needed to furnish a unique solution of eq.(11) we may say that this equation yields a unique solution for the SFT action. It is, however, also possible that additional information will be necessary. It is obviously an interesting question to find the minimal extra input needed to obtain from the RG equations (9-11) a unique solution for the SFT action. In that, insight obtained from matrix models and low dimensional string theories should be useful.

\section{World-sheet and space-time RG equation}

We proceed to demonstrate how eq.(10) is related to a world-sheet RG equation. The role of conformal invariance in SFT, as well as the issue of background independence were also discussed in ref.[25].

A world-sheet RG equation states that the total derivative of the effective action $\Gamma$ with respect to the world-sheet cutoff vanishes, as the cutoff is removed.

$$
\frac{d \Gamma}{d \log \epsilon}=\frac{\partial \Gamma}{\partial \log \epsilon}+\frac{\delta \Gamma}{\delta g_{A}} \frac{\partial g_{A}}{\partial \log \epsilon}=0
$$

where the $g_{A}$ are the bare two dimensional couplings, i.e. the background fields defining the underlying Sigma model. $\Gamma$ may be thought of either as the connected vacuum amplitude in the curved background, $\Gamma=-i T^{0}$, or as the generating functional for connected string amplitudes in a flat background.

Eq.(13) is the statement of renormalizability of the theory: All dependence on the cut-off may be absorbed in a running of the bare couplings. We may of course write down similar world-sheet RG equations for any N-point amplitude. These will involve also the scaling behaviour induced by the dimensions $\Delta_{A}$ of the integrated vertex operators. The on-shell condition is $\Delta_{A}=0$. 
The theory is independent of the cut-off ( $\sim$ the two-dimensional metric) if and only if we are at a fixed point

$$
\beta\left(g_{A}\right) \equiv \frac{\partial g_{A}}{\partial \log \epsilon}=0
$$

In this case the world-sheet RG equation is satisfied on account of each term on the r.h.s. of eq.(13) vanishing individually. This is then a fixed point solution of the world sheet RG equation.

The fixed point conditions (14) can be thought of as a set of consistency conditions. If we are not at a fixed point, the string theory will have to be modified to incorporate the propagating Liouville mode.

Next, consider the space-time RG equation, given by eqs.(10-12). It holds for any value of $a \geq \epsilon$, and for all $N \geq 0$. In general, for $a>\epsilon$, we have

$$
\begin{aligned}
& (-i)^{N} T^{G, N}\left(A_{1}, \ldots, A_{N} ; g_{A}(\epsilon) ; \epsilon\right)= \\
& \mathcal{V}_{A_{1}, \ldots, A_{N}}^{G, N}\left(g_{A}(\epsilon) ; a\right)+R_{A_{1}, \ldots, A_{N}}^{G, N}\left(g_{A}(\epsilon) ; a ; \epsilon\right)
\end{aligned}
$$

$R^{G, N}$ is the part of the amplitude corresponding to that part of moduli space covered by sewing together more elementary vertices with the propagator. Since the propagator vanishes for $a=\epsilon$ we have $R^{G, N}(a, a)=0, R^{G, N}$ covers a vanishing domain of N-punctured G-loop moduli space in this limit.

The recursion relation (11) states the independence of $T^{G, N}$ on $a$ (for $(G, N) \neq$ $(0,2))$ :

$$
0=\frac{\partial \mathcal{V}^{G, N}}{\partial \log a}+\frac{\partial R^{G, N}}{\partial \log a}
$$

where we dropped some of the indices for transparency. We are interested in the dependence of $T^{G, N}$ on the world-sheet cut-off

$$
(-i)^{N} \frac{\partial T^{G, N}}{\partial \log \epsilon}=\frac{\partial R^{G, N}}{\partial \log \epsilon}
$$

Taking $a=\epsilon$ in eq.(15) we arrive at

$$
(-i)^{N} \frac{\partial T^{G, N}}{\partial \log \epsilon}=\left.\frac{\partial \mathcal{V}^{G, N}}{\partial \log a}\right|_{a=\epsilon}
$$

Eq.(18) is the basic equation that relates the world-sheet and the space-time RG equation. 
For a fixed point solution of the world-sheet RG equation (13) we have $\partial T^{N} / \partial \log \epsilon=0$ on-shell. Eq.(18) then states that also

$$
\left.\frac{\partial}{\partial \log a} \mathcal{V}^{N}\right|_{a=\epsilon}=0
$$

on-shell, i.e. a fixed point solution of the world-sheet $R G$ equation is automatically a fixed point solution of the space-time $R G$ equation.

This is not too surprising: Any dependence of the full on-shell amplitudes on $a$ would certainly violate conformal invariance.

The opposite statement is less obvious. If we have a fixed point solution of the space-time RG equation, eq.(19) holds on-shell for all values of $N \geq 0$. It is then an open question whether one can derive from eq.(11) all of the conditions (14). Rather than to attempt solving this general problem we consider in the following sections the infrared limit $a=\epsilon \rightarrow 0$ where all massive string states are integrated out. In this case the general constraint (14) is known to be equivalent to 1) the vanishing of the corresponding tadpole amplitude and 2) the equations of motion for the background [16].

\section{World Sheet RG Equation in Flat Space}

We proceed to identify the space-time fixed point condition in the limit $a=\epsilon \rightarrow$ 0 . This is the ultraviolet limit on the world-sheet but the infrared limit in the space-time. We use eq.(11) to identify $\partial T^{N} / \partial \log \epsilon$ directly in terms of the Green's functions themselves.

In the recursion relation (11) appears

$$
\left.\frac{\partial \Delta^{A B}}{\partial \log a}\right|_{a=\epsilon}=-i \frac{\alpha^{\prime}}{2} \epsilon^{L_{0}+\bar{L}_{0}} \delta_{L_{0}, \bar{L}_{0}}<A^{*}\left|\bar{b}_{0} b_{0}\right| B^{*}>
$$

This vanishes as a power of $\epsilon$ for $\epsilon \rightarrow 0$, except for states with $L_{0}+\bar{L}_{0}=0$. The explicit $\epsilon$-dependence is therefore due to propagating states with $L_{0}+\bar{L}_{0}=0$.

To proceed beyond this point it is necessary to make a specific assumption for the background. We start with a flat background, i.e. the target space metric $G_{\mu \nu}$ and the dilaton field $\Phi$ are taken to be constant in space and time. All other background fields are taken to be zero.

In this case we have momentum conservation and

$$
L_{0}=\frac{\alpha^{\prime}}{4} p^{2}+\sum_{n=1}^{\infty} n a_{-n}^{\mu} a_{n}^{\nu} G_{\mu \nu}+\sum_{n=1}^{\infty} n\left(c_{-n} b_{n}+b_{-n} c_{n}\right)-1
$$


If the external states are taken conveniently off-shell, there is only one situation where an internal on-shell propagation always occurs: The case of tadpole diagrams, where the exchanged momentum is zero by momentum conservation alone. There are only two propagating states with $L_{0}+\bar{L}_{0}=0$ for vanishing momentum which give rise to tadpole diagrams: The trace of the graviton

$$
\epsilon_{\mu \nu} a_{-1}^{\mu} \bar{a}_{-1}^{\nu} \mid q=1, \bar{q}=1>
$$

and the dilaton

$$
\frac{1}{\sqrt{2}}\left(b_{-1} \bar{c}_{-1}+c_{-1} \bar{b}_{-1}\right) \mid q=1, \bar{q}=1>
$$

having

$$
\left.\frac{\partial \Delta^{A B}}{\partial \log a}\right|_{a=\epsilon}=\mp i \frac{\alpha^{\prime}}{2} \delta^{A B}
$$

respectively.

The normalization is such that for propagating states in the ghost vacuum

$$
\lim _{\substack{a \rightarrow 1 \\ \epsilon \rightarrow 0}} \Delta^{A B}=\frac{-i}{p_{A}^{2}+m_{A}^{2}} \delta^{A B}
$$

Integrating out the ghosts leads to the following vertex operator insertions $[26,20$, 21]

$$
\begin{aligned}
\text { graviton } & \sim \frac{\kappa}{2 \pi \alpha^{\prime}} \epsilon_{\mu \nu} \int d^{2} \xi \sqrt{g}: \partial_{a} X^{\mu} \partial^{a} X^{\nu}: \\
\text { dilaton } & \sim \frac{\kappa}{4 \pi \sqrt{2}} \int d^{2} \xi \sqrt{g} R^{(2)}
\end{aligned}
$$

Here $\kappa$ is the gravitational coupling, related to Newton's constant by $\kappa^{2}=8 \pi G_{N}$, and the polarization is normalized by $\epsilon_{\mu \nu} \epsilon^{\mu \nu}=1$.

$R^{(2)}$ is the curvature on the world sheet, related to the Euler character of the Riemann surface, $\chi$ by

$$
\chi \equiv 2-2 G=\frac{1}{4 \pi} \int d^{2} \xi \sqrt{g} R^{(2)}
$$

The propagators appearing inside loops do not present a divergent $\epsilon$ dependence, since the singularity of the propagator on-shell is removed by the loop momentum integration measure for $D>2$ space-time dimensions. Note that because of that the linear term on the r.h.s of eq.(10) drops out and we remain with the quadratic term only. 
The tachyon singularities can be removed by analytical continuation in the tachyon mass [27]. This introduces a complex value for the partition function at each genus $G \geq 1$ : The vacuum configuration of zero tachyon field is unstable, simply because the mass squared is negative. However, this problem would not appear in a string theory with world sheet supersymmetry and is physically quite distinct from that of the dilaton tadpole.

Therefore, combining eqs.(18) and (11) with $N=0$ we arrive at

$$
\begin{aligned}
& \frac{\partial T^{0}}{\partial \log \epsilon}=\left.\frac{\partial \mathcal{V}^{0}}{\partial \log a}\right|_{a=\epsilon}= \\
& \quad \frac{-i \alpha^{\prime}}{4} \sum_{G_{1}, G_{2}}\left[T^{G_{1}, 1} \text { (graviton) } T^{G_{2}, 1}\right. \text { (graviton) } \\
& \left.\quad-T^{G_{1}, 1} \text { (dilaton) } T^{G_{2}, 1} \text { (dilaton) }\right] \\
& \quad+\text { terms vanishing as } \epsilon \rightarrow 0
\end{aligned}
$$

Similarly, for $N=1$ we obtain

$$
\begin{aligned}
\frac{\partial T^{1}}{\partial \log \epsilon}(A)= & \frac{-i \alpha^{\prime}}{2} \sum_{G_{1}, G_{2}}\left[T^{G_{1}, 2}(A, \text { graviton }) T^{G_{2}, 1}\right. \text { (graviton) } \\
& \left.-T^{G_{1}, 2}(A, \text { dilaton }) T^{G_{2}, 1}(\text { dilaton })\right] \\
& + \text { terms vanishing as } \epsilon \rightarrow 0
\end{aligned}
$$

where the state $A$ can be both a graviton and a dilaton. As for $N=0$ all states are strictly on-shell by momentum conservation.

The correct fixed point condition for the SFT RG equation in the limit $a=\epsilon \rightarrow 0$ is that

$$
\frac{\partial \mathcal{V}^{0}}{\partial \log a}=0 \quad \text { and } \quad \frac{\partial \mathcal{V}_{A}^{1}}{\partial \log a}=0
$$

Although each of these equations individually may have non-trivial solutions (q.v. ref.[11] in the case $N=0$ ), the only solution that satisfies all of them is the vanishing of both massless tadpoles

$$
\sum_{G} T^{G, 1}(\text { graviton })=\sum_{G} T^{G, 1}(\text { dilaton })=0
$$

that is, we arrive at the fixed point condition for the world-sheet RG equation. These are also the equations of motion for the background. 
Since tree-level tadpoles vanish in a flat background, the summation in eq.(31) is over $G_{1}, G_{2} \geq 1$. The 1-point amplitudes may be expressed in terms of the dilaton potential defined by

$$
V(\Phi)=\sum_{G=1}^{\infty} V_{G}(\Phi) \equiv \frac{i}{\mathrm{Vol}} \sum_{G=1}^{\infty} T^{G, 0}\left(G_{\mu \nu}, \Phi\right)
$$

by using the relations [21]

$$
\begin{aligned}
\int^{\Sigma_{G}} e^{-S_{S M}(\Sigma)} \int d^{2} \xi \sqrt{g(\xi)} R^{(2)}(\xi) & =-4 \pi \chi_{G} V_{G}(\Phi) \mathrm{Vol} \\
\int^{\Sigma_{G}} e^{-S_{S M}(\Sigma)} \int d^{2} \xi \sqrt{g(\xi)}: \partial_{a} X \cdot \partial^{a} X(\xi): & =-\alpha^{\prime} \pi D\left(\chi_{G}-2\right) V_{G}(\Phi) \mathrm{Vol}
\end{aligned}
$$

Here

$$
S_{S M}=\frac{1}{4 \pi \alpha^{\prime}} \int d^{2} \xi \sqrt{g} \partial_{a} X^{\mu} \partial^{a} X^{\nu} G_{\mu \nu}+\frac{1}{4 \pi} \int d^{2} \xi \sqrt{g} R^{(2)} \Phi+\ldots
$$

is the Sigma model action and $\mathrm{Vol}=(2 \pi)^{D} \delta^{D}(p=0)$ is the volume of space-time.

We have introduced the abbreviated notation

$$
\int^{\Sigma_{G}} \equiv \int^{\Sigma_{G}} \mathcal{D} X \quad d^{3 G-3} \Omega \wedge d^{3 G-3} \bar{\Omega}
$$

The matter field $X$ is path integrated over the genus $\mathrm{G}$ Riemann surface $\Sigma_{G}$ for a fixed configuration of the Liouville field. $\Omega$ is the measure on moduli space. It is understood that we also divide by the infinite volume of the projective group, in accordance with the prescription of ref.[28].

Finally, using

$$
\chi_{G} V_{G}(\Phi)=-\frac{d}{d \Phi} V_{G}(\Phi) \equiv-V_{G}^{\prime}(\Phi)
$$

and formally summing over all genera we may rewrite eqs.(31) on the form

$$
2 V(\Phi)+V^{\prime}(\Phi)=V(\Phi)=0
$$

with the obvious solution $V=V^{\prime}=0$. This has the following unsurprising content: Flat space is a consistent background for the bosonic string for an arbitrary value of the dilaton field if and only if the dilaton potential is identically vanishing. 


\section{Non-flat backgrounds and the Fischler -Suss- kind Mechanism}

In this section we extend the analysis of the previous section to the more general case of a curved background. To evaluate the SFT RG equation in the limit $a=\epsilon \rightarrow 0$ in a general non-flat background is very hard. We need to know a factorization formula similar to eqs.(28),(29) for an interacting 2-dimensional conformal field theory. We therefore consider backgrounds with small curvature, where we still have an approximate factorization formula. We demonstrate how to extract from the space-time fixed point condition the Fischler-Susskind background shift (to first order in $\alpha^{\prime}$ and $\left.\log \epsilon\right)$.

We may obtain the effective action in a curved background by substituting

$$
\begin{aligned}
G_{\mu \nu} & \rightarrow G_{\mu \nu}(X) \\
\Phi & \rightarrow \Phi(X)
\end{aligned}
$$

in eq.(34).

We expand in powers of $\alpha^{\prime} / R^{2}$ (where $R$ is the radius of curvature of the background) by introducing Riemann normal coordinates [29] around an arbitrary point $x$ :

$$
\begin{aligned}
G_{\mu \nu}(X) & =G_{\mu \nu}(x)-\frac{1}{3} R_{\mu \lambda \nu \kappa}(x) X^{\lambda} X^{\kappa}+\mathcal{O}\left(\frac{1}{R^{3}}\right) \\
\Phi(X) & =\Phi(x)+\nabla_{\mu} \Phi(x) X^{\mu}+\frac{1}{2} \nabla_{\mu} \nabla_{\nu} \Phi(x) X^{\mu} X^{\nu}+\mathcal{O}\left(\frac{1}{R^{3}}\right)
\end{aligned}
$$

The zeroth order approximation corresponds to the flat background considered above. Our conventions for the Ricci tensor follow those of ref.[29].

To first order in $\alpha^{\prime}$ one may still neglect the effects of curvature in the factorization of loop tadpoles, since the factorized expression in flat space is by itself proportional to $\alpha^{\prime}$ (q.v. eq.(24)). This means that the eqs.(28,29) remain valid and hence the fixed point condition for the SFT RG equation implies again the vanishing of the tadpole amplitudes. We proceed to demonstrate how this leads to the equations of motion for the non-flat background, repeating the analysis of ref.[14, 21] in our framework.

The new feature of the curved background is that the tadpole at tree-level no longer vanishes. We may represent the tree-level graviton and dilaton tadpoles by 
a shift in the corresponding background fields [14]:

$$
\begin{aligned}
\Phi^{0} & =\Phi+\delta \Phi \\
G_{\mu \nu}^{0} & =G_{\mu \nu}+\delta G_{\mu \nu}
\end{aligned}
$$

where to lowest order in $\log \epsilon$ we simply have [16]

$$
\begin{aligned}
\delta \Phi(x) & =-\left(\frac{1}{2} \alpha^{\prime} \square \Phi(x)-\alpha^{\prime}(\nabla \Phi(x))^{2}\right) \cdot \log \epsilon+\mathcal{O}\left(\frac{\alpha^{\prime}}{R^{2}}\right) \\
\delta G_{\mu \nu}(x) & =\left(\alpha^{\prime} R_{\mu \nu}(x)+2 \alpha^{\prime} \nabla_{\mu} \nabla_{\nu} \Phi(x)\right) \cdot \log \epsilon+\mathcal{O}\left(\frac{\alpha^{\prime}}{R^{2}}\right)
\end{aligned}
$$

We define the 2-dimensional quantum field theory formally given by eq.(34) by dimensional regularization (DR) on the world-sheet. We still take all vertex operators to be defined through oscillator normal ordering. As shown in ref. [21] the two regularizations are related by

$$
: \partial_{a} X^{\mu} \partial^{a} X^{\nu}:=\left(\partial_{a} X^{\mu} \partial^{a} X^{\nu}\right)_{D R}+\frac{\alpha^{\prime}}{4} G^{\mu \nu} R^{(2)}
$$

We may rewrite the action of the sigma model

$$
\begin{aligned}
S_{S M}= & \frac{1}{4 \pi \alpha^{\prime}} \int d^{2} \xi \sqrt{g}: \partial_{a} X^{\mu} \partial^{a} X^{\nu}: G_{\mu \nu}^{0} \\
& +\frac{1}{4 \pi} \int d^{2} \xi \sqrt{g} R^{(2)} \phi^{0}+\ldots
\end{aligned}
$$

where we introduced

$$
\phi^{0}=\Phi^{0}-\frac{1}{4} G^{\mu \nu} G_{\mu \nu}^{0}
$$

in order to have background fields that diagonalize the propagator (q.v. eq.(26)).

The bare graviton 1-point tree amplitude is given by

$$
\begin{aligned}
& T^{0,1} \text { (graviton) }= \\
& \quad i \int^{\Sigma_{0}} \frac{\kappa}{2 \pi \alpha^{\prime}} \epsilon_{\mu \nu} \int d^{2} \xi \sqrt{g}: \partial_{a} X^{\mu} \partial^{a} X^{\nu}: e^{-S_{S M}}
\end{aligned}
$$

Expanding the exponential to first order in $\delta G_{\mu \nu}$ and $\delta \phi$ we obtain a term proportional to $\delta G_{\mu \nu}$ containing two vertex operator insertions. To lowest non-vanishing order in $\alpha^{\prime}$ we may then ignore the counterterm in the action. We then simply have the Polyakov path integral over the sphere in a flat background with two vertex operator insertions. This is nothing but the inverse propagator, this contribution is therefore $\sim \delta G_{\mu \nu} / \log \epsilon$ and hence finite in the limit $\epsilon \rightarrow 0$. 
This way one arrives at

$$
\begin{aligned}
T^{0,1} \text { (graviton) } & =\frac{i}{\alpha^{\prime} \kappa} \int \frac{\delta G^{\mu \nu}}{\log \epsilon} \epsilon_{\mu \nu} d^{D} x \sqrt{-G(x)}+\ldots \\
T^{0,1} \text { (dilaton) } & =-\frac{2 i \sqrt{2}}{\alpha^{\prime} \kappa} \int \frac{\delta \phi}{\log \epsilon} d^{D} x \sqrt{-G(x)}+\ldots
\end{aligned}
$$

Proceeding to evaluate the terms with $G \geq 1$ by the same methods as before, and summing up over $G$, we arrive at

$$
\begin{aligned}
T^{1} \text { (graviton) } & =\frac{i}{\alpha^{\prime} \kappa} \epsilon^{\mu \nu} \int\left\{\frac{\delta G_{\mu \nu}}{\log \epsilon}+\frac{\alpha^{\prime} \kappa^{2}}{2}\left(2 V+V^{\prime}\right) G_{\mu \nu}\right\} \cdot d^{D} x \sqrt{-G(x)} \\
T^{1} \text { (dilaton) } & =-i \frac{2 \sqrt{2}}{\alpha^{\prime} \kappa} \int\left\{\frac{\delta \phi}{\log \epsilon}-\frac{\alpha^{\prime} \kappa^{2}}{4} V^{\prime}\right\} \cdot d^{D} x \sqrt{-G(x)}
\end{aligned}
$$

Combining eqs. $(47,41)$, we arrive at the equations of motion

$$
\begin{aligned}
R_{\mu \nu}+2 \nabla_{\mu} \nabla_{\nu} \Phi & =-\frac{1}{2} \kappa^{2}\left(V^{\prime}(\Phi)+2 V(\Phi)\right) G_{\mu \nu} \\
\frac{1}{2} \square \Phi-(\nabla \Phi)^{2} & =-\frac{1}{8} \kappa^{2}\left((D-2) V^{\prime}(\Phi)+2 D V(\Phi)\right)
\end{aligned}
$$

These are exactly the equations of motion derived from the space-time effective action

$$
\Gamma=\frac{\lambda^{2}}{16 \pi G_{N}} \int d^{D} x \sqrt{-G} e^{-2 \Phi}\left(R+4(\nabla \Phi)^{2}\right)-\int d^{D} x \sqrt{-G} V(\Phi)
$$

in agreement with the analysis of ref.[21].

\section{Conclusions}

We have found that the space-time RG equation of string field theory is inextricably linked to the world-sheet RG equation of perturbative string theory. In the case of massless states we find that the fixed point conditions of the space-time equation imply the fixed-point conditions of the world-sheet equations, and vice versa. This mutual fixed point condition is nothing but the equations of motion for the massless background. So we have gone through a full circle, space-time, world sheet, spacetime. It is tempting to suggest that this relation holds in general, i.e. for general backgrounds and without any approximation, however we cannot prove that at this stage. 
The RG equation (10) seems to be a compact way of storing a lot of information about string theory. In this paper we showed that it, in some sense, contains world sheet RG equation. We also know that it contains the B-V equation. It would be interesting to find the analogue equation for matrix models and low dimensional solvable string theories. We could then see if this equation and its solutions indeed contains all the information on the theory or there are some other equations or conditions that form a complete set of equations for the theory.

A special feature of the approximation we used is that the double derivative linear term in the RG equation (10) was neglected. This linear term is certainly present in the B-V equation, i.e. in the limit $a \rightarrow 1$. It's presence is expected to change the nature of the solution in a substantial way. In particular this term may be responsible for the anomalous growth of perturbative amplitudes in string theory [30]. To see if that is indeed correct a comparison with the B-V equation in ordinary gauge theories and open SFT may be useful.

The equations that we obtained are non-perturbative in the sense that they involve the dilaton potential $V$ obtained by summing over all genera. They do not, however determine the shape of the dilaton potential, only the background field configuration consistent with the energy-momentum tensor implied by $V$. In the framework of our analysis we seem to need some extra input to actually determine the effective action. In that respect, these equations seem to be weaker than the combination of string and flow equations in matrix models. If that is an artifact of our approximation remains to be seen.

It is entirely possible that the combination of space-time covariance and string symmetries that seem to work in the low-dimensional models are inadequate for the critical string where the physical degrees of freedom are enormously enriched. In any event the string field theory RG equation is an interesting non-perturbative constraint on the theory whose actual content deserves further investigation.

Acknowledgements. We would like to thank S. De Alwis, W. Fischler, J. Gaite, J. Polchinski and G. Veneziano for discussions. One of us (K.R.) would like to thank the Theory Group at the University of Texas for hospitality. 


\section{References}

[1] D. Gross and A. Migdal, Phys. Rev. Lett.64 (1990) 127.

M. Douglas and S. Shenker, Nucl. Phys.B335 (1990) 635.

E. Brezin and V. Kazakov, Phys. Lett.236B (1990) 144.

[2] L. Alvarez-Gaumé, Random Surfaces, Statistical Mechanics and String Theory, CERN preprint, CERN-TH.6061/91.

[3] E. Witten, Nucl. Phys.B340 (1990) 281.

[4] E. Verlinde and H. Verlinde, Nucl. Phys.B348 (1991) 457;

R. Dijkgraaf, E. Verlinde and H. Verlinde, Nucl. Phys.B348 (1991) 435.

[5] J. Polchinski, Texas preprint, UTTG-39-90.

[6] S. Das and A. Jevicki, Mod. Phys. Lett.A5 (1990) 1639.

J. Polchinski, Nucl. Phys.B346 (1990) 253.

D. Minic, J. Polchinski and Z. Yang, Texas preprint, UTTG-16-91.

[7] R. Brustein and S.P. De Alwis, Phys. Lett.247B (1990) 31.

[8] H. Sonoda and B. Zwiebach, Nucl. Phys.B331 (1990) 592;

Nucl.Phys.B336 (1990) 185 and references therein.

[9] T. Kugo, H. Kunitomo and K. Suehiro, Phys. Lett.226B (1989) 48.

[10] B. Zwiebach, MIT preprints, CTP-1830, CTP-1831, CTP-1872.

[11] R. Brustein and S.P. De Alwis, Nucl. Phys.B352 (1991) 451.

[12] J. Polchinski, Nucl. Phys.B231 (1984) 269.

[13] R. Brustein, D. Nemeschansky and S. Yankielowicz, Nucl. Phys. B301 (1988) 224.

I. Klebanov and L. Susskind, Phys. Lett. 200B (1988) 446.

J. Hughes, J. Liu and J. Polchinski, Nucl. Phys. B316 (1989) 15.

[14] W. Fischler and L. Susskind, Phys. Lett.171B (1986) 383;

Phys. Lett.173B (1986) 262. 
[15] C. Callan, D. Friedan, E. Martinec and M. Perry, Nucl. Phys.B262 (1985) 593.

[16] A.A. Tseytlin, Int. Jour. Mod. Phys.A5 (1990) 589;

A.A. Tseytlin, Sigma Models and Renormalization of String Loops, Lectures at the 1989 Trieste Spring School on Superstrings.

[17] R. Brustein and S.P. De Alwis, Texas preprint, UTTG-08-91 and in preparation.

[18] E. Witten, Nucl. Phys.B268 (1986) 253.

[19] D. Friedan, E. Martinec and S. Shenker, Nucl.Phys.B271 (1986) 93.

[20] E. Fradkin and A.A. Tseytlin, Nucl.Phys.B261 (1985) 1;

S.P. de Alwis, Phys.Lett.168B (1986) 59;

C. Callan and Z. Gan, Nucl.Phys.B272 (1986) 647

[21] J. Polchinski, Nucl. Phys.B307 (1988) 61.

[22] A. Le Clair, Nucl.Phys.B303 (1988) 189.

[23] C. Itzykson and J.-B. Zuber, Quantum Field Theory, McGraw-Hill (1980).

[24] B. Zwiebach, MIT preprint, CTP-1926.

[25] A. Sen, Phys. Lett.241B (1990) 350;

Nucl. Phys.B345 (1990) 551;

Nucl. Phys.B347 (1990) 270.

[26] S. Weinberg, Phys.Lett.156B (1985) 309.

[27] N. Marcus, Phys.Lett.219B (1989) 265.

[28] A.A. Tseytlin, Phys.Lett.208B (1988) 221.

[29] C.W. Misner, K.S. Thorne and J.A. Wheeler, Gravitation (Freeman, San Francisco, 1972).

[30] S. Shenker, Rutgers preprint, RU-90-47. 